Wunderlich, Philipp; Größler, Andreas; Zimmermann, Nicole; Vennix, Jac (2014): Managerial Influence on the Diffusion of Innovations within Intra-organizational Networks, System Dynamics Review, Vol. 30, No. 3, pp. 161-185.

\title{
Managerial Influence on the Diffusion of Innovations within Intra- organizational Networks
}

\author{
Philipp Wunderlich, Andreas Größler, Nicole Zimmermann and Jac A. M. Vennix
}

\begin{abstract}
This study analyzes the dynamics of intra-organizational innovation implementation processes based on conversion within and migration between groups. We employ a diffusion model that extends often-used epidemic models in three ways: (1) repeated acceptance and rejection decisions of adopters and non-adopters, (2) diffusion within and across groups organized in different network structures and (3) management's continuous influence on the diffusion process. The model is used to identify and evaluate senior management's possibilities to steer the diffusion process. Analyses reveal that network structure influences the interplay between the self-reinforcing dynamics of conversion and the balancing dynamics of migration. Additionally, management can minimize diffusion time and invested resources by considering the proximity of groups to each other and to the network periphery. Providing structural explanations, this study offers insights into why, given various intra-organizational networks, management policies can yield different results on whether and how innovations are adopted in an organization.
\end{abstract}

Keywords: networks, intra-organizational innovation diffusion, communication structure, migration, conversion 


\section{Introduction}

Organizations regularly innovate internally. For instance, they introduce new IT systems, establish Total Quality Management (TQM) in manufacturing plants, or implement regulations to counter fraud and other professional misconduct. Such intraorganizational innovations, no matter whether they concern products or processes, are for many companies as important as a new product launch because a company can remain competitive only if it implements innovations that create value efficiently. The success of such intra-organizational implementation processes depends on the continuous decisions of organizational members to use the innovation (Choi and Chang, 2009; Venkatesh et al., 2003). However, the implementation of innovations in organizations frequently fails. That is, new products and processes are often not used as desired by management. For example, Aiman-Smith and Green (2002, p. 421) state that a 47 percent failure rate of new technology implementations "is a major concern of U.S. manufacturing managers and researchers." Similarly, Chen et al. (2009) mention a survey which found that 40 percent of enterprise resource planning projects failed to meet the business case. Unsuccessful implementation efforts not only waste time and resources, but might even jeopardize organizational survival. In order to remain competitive, implementing new practices successfully and promptly is crucial for organizations in rapidly changing industries, such as telecommunications and media and entertainment.

Since intra-organizational innovation implementation depends on employees' individual adoption decisions (Lanzolla and Suarez, 2012; Leonard-Barton and Deschamps, 1988), it resembles the diffusion of a product or service innovation in a market. Therefore, similar methods can be used to analyze these processes. However, in contrast to market-oriented diffusion models (Bass, 1969), models of intraorganizational innovation diffusion should account for (1) employees alternating between using and neglecting the innovation (Ulli-Beer et al., 2010), (2) employees' heterogeneity with respect to their team or department affiliation (Hansen, 1999; Tsai, 2001), and (3) management's efforts to make the innovation successful (Choi and Chang, 2009; Repenning, 2002). Therefore, this paper introduces a model of intraorganizational innovation diffusion dynamics. While based on classical diffusion studies from the field of system dynamics (e.g., Milling, 1996; Maier, 1998), it extends these by the three characteristics listed above (which are discussed further in the next section). The purpose of this study is to identify successful management strategies which ensure a complete diffusion within different intra-organizational network structures.

The structure of the paper is as follows. After a short literature review in section two, we describe the system dynamics model that is partially inspired by an algebraic model of innovation diffusion in intra-organizational networks (Krackhardt, 1997). Simulation analyses in the fourth section focus not only on outcomes but also on the diffusion process itself and its underlying dynamics. In section five, we analyze the normative influence of senior management on organizational implementation processes and derive principles of successful implementation strategies that are applicable to various network structures. The paper closes by summarizing and discussing the derived insights and by outlining possible further research. 


\section{Characteristics of innovation diffusion in organizations}

The organizational implementation phase, as the critical period between the decision to adopt and the routine usage of an innovation (Klein and Sorra, 1996; Rogers, 2003), has received increasing attention by scholars. Research has identified deficient implementation processes as a major reason for organizations' failure to benefit from adopted innovations (Klein and Sorra, 1996; Aiman-Smith and Green, 2002; Gary, 2005; Karimi et al., 2007). Despite the growing number of studies that identify multiple causes of unsuccessful implementation processes, literature lacks multidimensional models that explain the difference between successful and unsuccessful implementation efforts. Such models should take into account multiple and to some extent interrelated drivers of implementation success (Dean Jr. and Bowen, 1994; Klein and Sorra, 1996; Klein et al., 2001; Repenning, 2002). Greenhalgh et al. (2005, p. 135) criticize existing literature for implicitly assuming that "the determinants of innovation can be treated as variables whose impact can be isolated and independently quantified." However, more recent work suggests that "in reality the different determinants of organizational innovativeness interact in a complex way with one another" (Greenhalgh et al., 2005, p. 135). Existing implementation studies barely focus on these interactions, in particular with regard to drivers of innovation diffusion on different organizational levels. Mostly, they focus either on employee-related processes, examining "employees' affective and behavioral responses to an innovation," or on organizational/institutional processes, focusing on the management support, structure, and resources of the implementing organization (Choi and Chang, 2009, p. 245).

A further limitation of many diffusion models is that only adopters exert social pressure on non-adopters, whereas non-adopters are assumed to have no influence on adopters. Therefore, adopters are assumed to never revise their adoption decision and reject a previously adopted innovation, whereas non-adopters are assumed to reconsider adopting it at frequent intervals (Abrahamson and Rosenkopf, 1997; Yücel and van Daalen, 2011). The assumption "once an adopter always an adopter" is justifiable for the purchase of an innovation, as addressed in marketing research. Durable goods are a prime example (Bass, 1969; Mahajan et al., 1990). However, in an intra-organizational context, an adopter is somebody who uses the innovation and not necessarily the individual that purchased it. Hence, the most crucial issue is the individual adoption decision of each employee to use the innovation instead of senior management's decision to purchase it (Choi and Chang, 2009; Leonard-Barton and Deschamps, 1988). This approach is supported by Mahajan et al. (1984) who point out that communicators not only promote an innovation but may also transfer neutral as well as negative information about it through word-of-mouth (Rogers, 2003). The exchange of this information plays an important role even after the adoption decision has been made because individuals seek reinforcement and may reverse this decision if they are exposed to conflicting messages about this innovation (Rogers, 2003). Thus, we consider positive as well as negative word-of-mouth within communication networks, meaning that the percentage of adopters of an innovation cannot only grow but also shrink.

Accounting for the influence of positive and negative word-of-mouth simultaneously implies that some individuals might alternate between adopting an innovation and discontinuing it. For example, an employee might use a newly 
implemented system to manage customer relationships if this person's team members communicate that they also use it (positive word-of-mouth). However, if the team members of the next project tell this employee that they prefer the status quo over the innovation (negative word-of-mouth) this employee might also decide to stop using the innovation, at least for this particular project. By considering the possibility that some employees might alternate between using and neglecting the innovation, the introduced model resembles SIS models which account for the possibility that individuals become again susceptible (S) to a disease after having been infected (I) with it (e.g., Dorogovtsev et al., 2008).

Organizational change-like the implementation of an innovation-“is created, sustained, and managed in and by communications" (Ford and Ford, 1995, p. 560). Donnellon (1986), for example, argues that the actual implementation of change is all about communication. Therefore, this article focuses on communication processes between organizational members. Other factors, such as the relative advantage of an innovation or employees' experiences (Rogers, 2003), are not considered. The few implementation studies that account for the interaction of employee-related and organizational processes (Damanpour, 1996; Gosselin, 1997; Repenning, 2002; Choi and Chang, 2009) acknowledge the communication between employees as a main driver of organizational change. However, they usually neglect the concrete structure of communication depicting which groups of employees interact with each other.

On the other hand, the communication relations between groups, which form an intra-organizational network, are considered to be essential in diffusion research (e.g., Abrahamson and Rosenkopf, 1997). Therefore, following Choi and Chang (2009), this paper brings together employee-related aspects (i.e., innovation acceptance and peer influence) and organizational aspects (i.e., network structure and management influence) in order to reveal the dynamics caused by intra-organizational communication networks and to analyze ways senior management can influence those dynamics to ensure an organization-wide implementation of the respective innovation. We aim at identifying characteristics of successful management strategies which consider the intra-organizational network structure.

Consequently, we extend the common structure of diffusion models in system dynamics by accounting for (1) repeated acceptance and rejection decisions of adopters and non-adopters which might cause employees to alternate between using the innovation (adopters) and the status quo (non-adopters), (2) the network structure between departments or teams, and (3) management's normative influence on the organizational diffusion process. Thereby, we go beyond a mere description of diffusion dynamics in order to explain how management can influence organizational diffusion processes among employees. Thus, the system dynamics model presented in this study addresses intra-organizational innovation implementation by combining employeerelated and organizational factors. In contrast to Choi and Chang (2009), we do not focus on the strength of empirical correlations between influencing factors. Instead, we address the network-caused dynamics between actors. This allows us to account for changes of influencing factors and to analyze effective and efficient managerial strategies for implementing innovations in organizations. 


\section{Modeling intra-organizational innovation diffusion}

Since "[a]ll innovations carry some degree of uncertainty for an individual, who is typically unsure of the new idea's functioning and thus seeks social reinforcement from others of his or her attitude toward the innovation" (Rogers, 2003, p. 175), social communication networks play an important role in the diffusion of organizational innovations. We analyze the innovation-related communication within intraorganizational networks by distinguishing between five homogeneous and equally large groups of employees. Within each group there is an adopter camp, consisting of employees who use the innovation, and a non-adopter camp, consisting of employees who neglect the innovation (see Fig. 1). These groups can represent, for example, worldwide branch offices of an enterprise or homogeneous departments of an organization which are connected to each other through communication, thereby forming a social network. We chose the group as the level of our analysis because senior management mostly influences the diffusion process on an aggregated rather than an individual level.

Building on Krackhardt's (1997) diffusion model, this section introduces a model of a communication network in which five groups of equal size are organized in a chain structure, as depicted in Fig. 1. By means of this structure, we illustrate the core dynamics of communication networks in the subsequent section. In the section thereafter, we derive principles that can help senior management to develop successful implementation strategies and we examine to what extent these principles apply to other network structures as well.

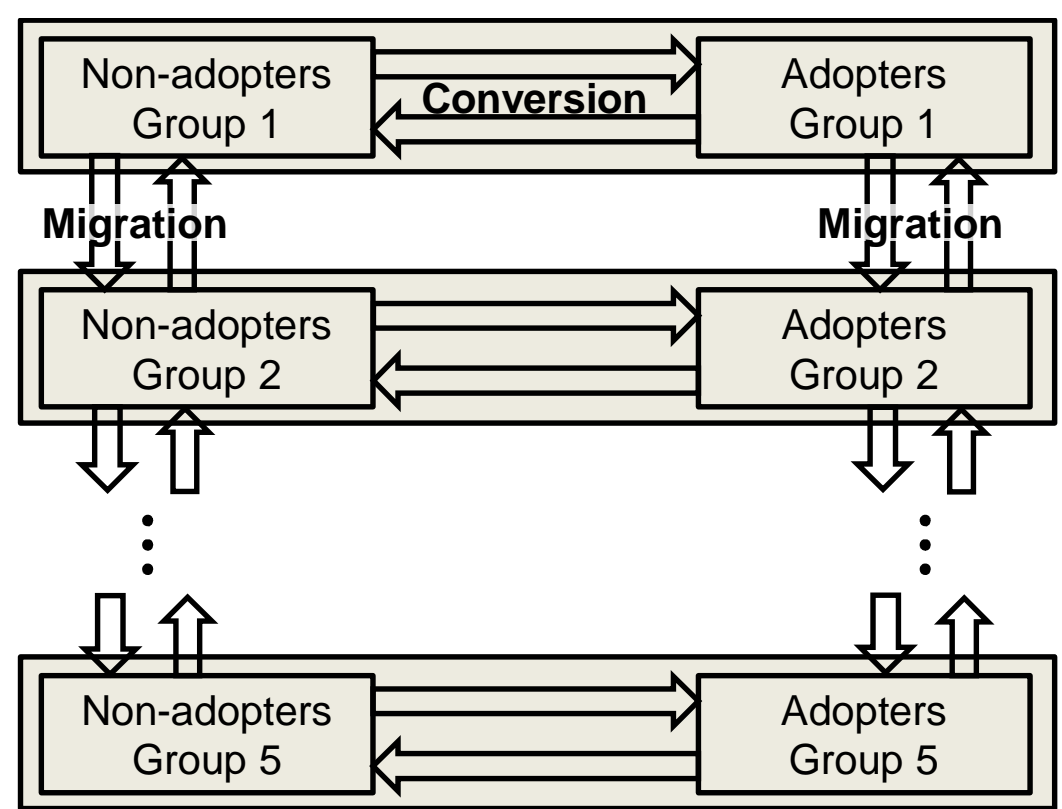

Fig. 1. Organizational innovation diffusion process within a five-membered chain structure.

Similar to Krackhardt (1997), we divided the communication process between adopters and non-adopters into two sub-processes. First, employees exchange opinions and experiences between groups. This so-called migration is modeled by exchanging a certain fraction of adopters as well as non-adopters with all connected groups. For example, in the five-membered chain structure depicted in Fig. 1, group 2 sends a 
certain fraction of its adopters to the adopter camps of the connected groups 1 and 3 , which, in return, send certain fractions of adopters to group 2. This migration also takes place between the non-adopter camps of connected groups. Second, adopters and nonadopters communicate with each other within a group, thereby trying to convince the other party (Wood et al., 1994; Krackhardt, 1997). In the course of this so-called conversion, a fraction of adopters as well as non-adopters is converted by the opposing party. The degree of diffusion within a group is measured by the proportion of adopters $A_{i}$ of a group $i$. The term $\left(1-A_{i}\right)$ represents the proportion of non-adopters because the proportions of adopters and non-adopters within a group $i$ always add up to one.

The stock and flow diagram, illustrated in Fig. 2, depicts the two stocks Fraction Adopters and Fraction Non-adopters and their conversion and migration rates. Since all groups are assumed to be structurally equal, Fig. 2 only depicts the model structure for one group, while all other groups are subscripted. The network structure between groups is defined by an adjacency matrix, represented by the parameter Is Connected To (see full model documentation in electronic supplement).

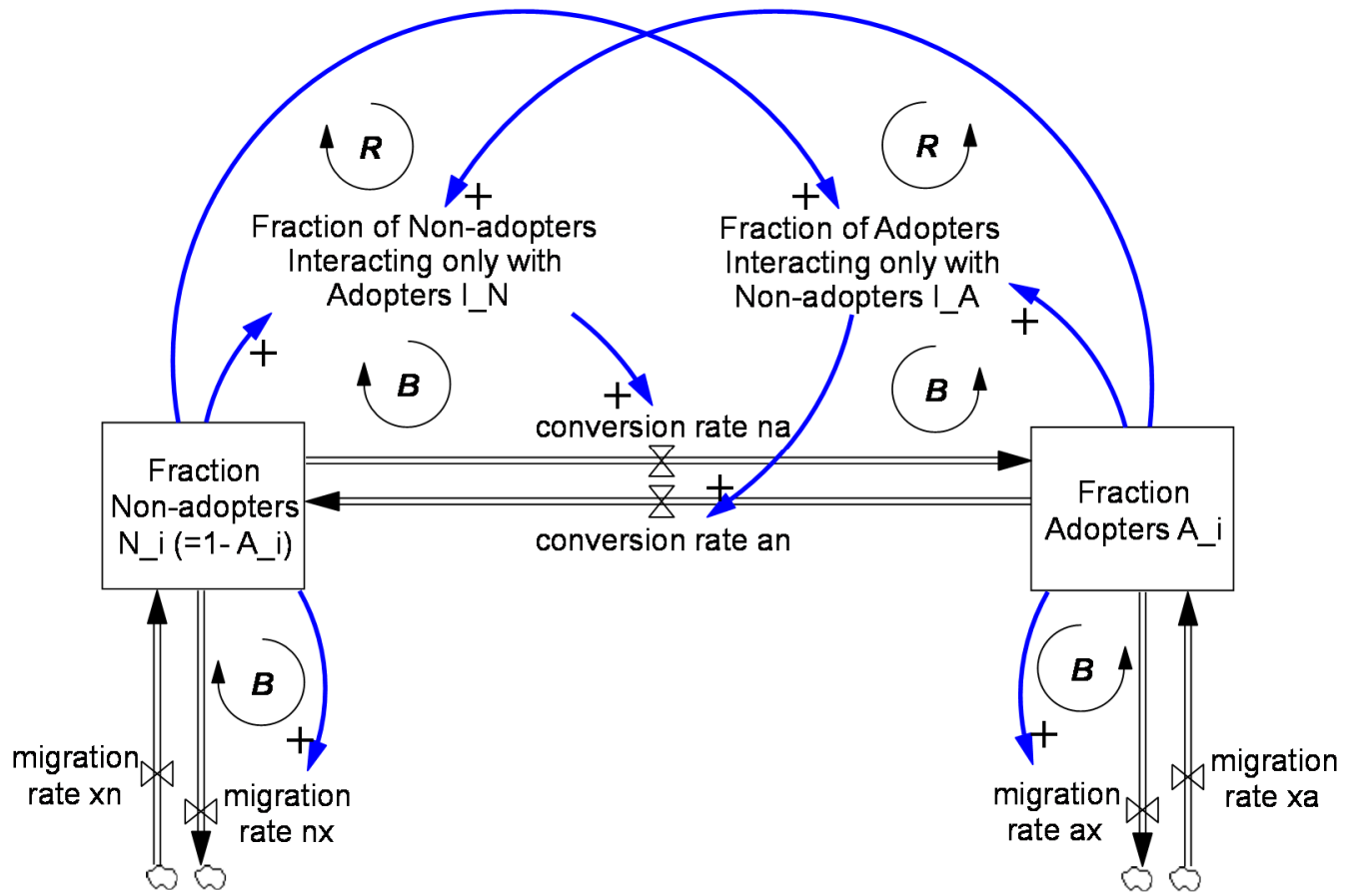

Fig. 2. Simplified stock and flow diagram of the migration and conversion processes (the five organizational groups are modeled with subscripts).

The lower part of Fig. 2 shows that a certain adopter as well as non-adopter fraction migrates into the respective camp of each connected group in the course of the migration process. Since we use day as the unit for time, the unit of the migration rate equals percent per day. The adopter as well as non-adopter fraction that leaves a group $i$ to migrate into a connected group $j$ depends on the size of the adopter or non-adopter camp within group $i$ and on the daily migration rate between group $i$ and group $j\left(m_{i j}\right)$. Equation 1 specifies the fraction of adopters emigrating out of group $i$ into all connected groups $j$ (migration rate ax in Fig. 2):

$$
\frac{d A_{\text {i emigr }}}{d t}=\sum_{j} A_{i} \cdot m_{i j}
$$


However, while adopters and non-adopters emigrate out of a group $i$, adopters and nonadopters from the connected groups $j$ immigrate into group $i$. Equation 2 specifies the amount of adopters immigrating into group $i$ (migration rate $x a$ in Fig. 2):

$$
\frac{d A_{i \text { immigr }}}{d t}=\sum_{j} A_{j} \cdot m_{j i} .
$$

The concept of migration can be extended to other forms of group-spanning communication such as making a telephone call or using an instant messenger service. For this purpose, we understand the migration rate between a group $i$ and a group $j$ $\left(m_{i j}\right)$ as the daily adopter or non-adopter fraction of group $i$ which initiates interactions with group $j$. Vice versa, the migration rate between group $j$ and group $i\left(m_{j i}\right)$ represents the daily adopter or non-adopter fraction of group $j$ which initiates interactions with group $i$. Thus, even though members of group $i$ do not need to migrate physically to bridge the distance to group $j$, they become part of group $j$ as soon as they start communicating with it. Thus, $m_{j i}$ also contains members of group $i$ that have only temporarily been part of group $j$ - for example for the duration of a telephone call—but then terminate their interaction with group $j$ and thereby become members of group $i$ again. Due to the conversion processes within groups, previous adopters of group $i$ might return as non-adopters, after communicating with group $j$. This interpretation of migration is possible because the continuous occurrence of migration and conversion allows organizational members to leave and return to a group within very short time intervals. Therefore, the proposed model is applicable in multiple cases, potentially providing insights into the dynamics of a variety of organizational communication networks that are not limited to physical migration processes between groups.

With regard to conversion, we follow Krackhardt (1997) who states that groupinternal communication is fuelled by employees' active search for innovation-related information, especially among peers. Prislin and Wood (2005, p. 677) argue that " $t]$ he views of other people are important in part because they help to structure the cacophony of stimuli to which we are regularly exposed, and thereby help us to operate among those stimuli." Referring to the work of Eagly and Chaiken (1993), they specify the influence of peers by stating that "others' attitudes impose structure and make sense out of the world by indicating whether objects are to be evaluated with some degree of favor or disfavor." (Prislin and Wood, 2005, pp. 677-678)

According to the concept of satisficing behavior, organizational members do not strive to obtain all information available from others (Simon, 1956). In line with Simon (1956), De Dreu et al. (2008, p. 25) emphasize that "people can and will choose among a shallow and heuristic versus a deep and deliberate information search-and-processing strategy." Therefore, we assume that organizational members randomly search for likeminded others only within a limited fraction of their group. Asch (1963, p. 186) found that the presence of only one other like-minded group member is "sufficient to deplete the power of the majority, and in some cases to destroy it." In accordance with Asch (1963), Schulz-Hardt et al. (2000, p. 659) state that "support by a second member gives the minorities additional self-confidence and thus increases their influence on the decision process." This bias towards a favored or chosen decision coincides with studies which found that information-seeking processes are often not balanced (Schulz-Hardt et al., 2000). That is, people prefer confirming over conflicting information (Janis and Mann, 1977; Frey, 1986; Prislin and Wood, 2005) and are therefore to a certain extent 
resistant to change (e.g., Kim and Kankanhalli, 2009). Consequently, we assume that an employee may convert to the opposite camp only if another like-minded organizational member cannot be found within the searched group segment. Isolated adopters convert to the non-adopter camp with a daily conversion probability of $P_{A N}$, while isolated nonadopters convert to the adopter camp with a daily conversion probability of $P_{N A}$.

In Fig. 2, the two flows linking the stock of adopters to the stock of non-adopters represent conversion (Ulli-Beer et al., 2010). Conceptually, we assume that adopters are more likely to convert status-quo oriented non-adopters than the other way around. This assumption is supported by East et al. (2008) who find that positive word-of-mouth has a bigger impact on brand purchase probability than negative word-of-mouth and by Berger and Milkman (2012, p. 201) who state: "While common wisdom suggests that people tend to pass along negative news more than positive news, our results indicate that positive news is actually more viral." Regarding the model, the greater influence of the innovation-affirming adopters translates into a higher search intensity of adopters $\left(S_{A}\right)$ than that of non-adopters $\left(S_{N}\right)$. The search intensities describe with how many other employees an employee interacts on average. Assuming that adopters use the innovation and spread positive word-of-mouth and that non-adopters do not use the innovation but spread negative word-of-mouth, $S_{A}$ and $S_{N}$ also indicate the strength of positive and negative word-of-mouth. That is, due to the higher strength of positive word-of-mouth, non-adopters are more easily converted by adopters than the other way around.

Equation 3 describes the daily non-adopter fraction of a group $i$ that converts to the adopter camp of that group because those non-adopters could not find any like-minded people within their searched group segment (conversion rate na in Fig. 2):

$$
\frac{d A_{\text {i conv }}}{d t}=P_{N A} \cdot I_{N}
$$

The fraction of isolated non-adopters only interacting with adopters $\left(I_{N}\right)$ corresponds to the term $I_{N}=\left(1-A_{i}\right) \cdot A_{i}{ }^{S_{N}}$, where $A_{i}{ }^{S_{N}}$ represents the probability that a nonadopter only meets adopters in his or her searched group segment. Within one day, these isolated non-adopters convert to the adopter camp with the conversion probability $P_{N A}$. The process works likewise for the conversion of adopters within a group $i$ (conversion rate an in Fig. 2):

$$
\frac{d N_{i c o n v}}{d t}=P_{A N} \cdot I_{A}
$$

Since the adopter fraction of a group $i$ depends on migration and conversion, the total increase of the adopter fraction of a group $i$ per day is defined as:

$$
\frac{d A_{i}}{d t}=\sum_{j} A_{j} \cdot m_{j i}-\sum_{j} A_{i} \cdot m_{i j}+P_{N A} \cdot I_{N}-P_{A N} \cdot I_{A}
$$

Regarding the model's internal validity (i.e., the logical consistency and formal correctness of the structure and parameters used in the model), various tests have been conducted: findings have been tested for very small time steps between iterations of calculation, resulting in no major changes in the dynamic behavior; dimensional consistency of the models has been secured; model robustness has been tested against extreme value conditions in the parameters (Forrester and Senge, 1980). With regard to external validity (i.e., the possibility to transfer derived insights to other relevant 
systems), one must consider the purpose of the model (Barlas, 1989; Barlas and Carpenter, 1990; Barlas, 1996). The purpose is to analyze the dynamics of innovation diffusion by relating structural determinants to the observed diffusion behavior. These dynamics will be shown and discussed in the remainder of this paper. In the following section, we consider intra-organizational innovation diffusion without, in the section thereafter, with management intervention.

\section{Dynamics within intra-organizational diffusion networks}

The simulations of the diffusion model in this section demonstrate under which conditions an adopter minority can convince a non-adopter majority within a fivemembered chain structure. With these simulations we illustrate the core dynamics of communication networks and demonstrate that the model is capable of reproducing Krackhardt's (1997) basic analyses before advancing our study to more elaborated policy tests. In the subsequent simulations, we assume that group 1 initially constitutes the adopter minority within the organization. Thus, group 1 serves as the mother group $\left(M G r_{1}\right)$ which is composed only of adopters, while the other four groups consist of nonadopters only. Such a peripheral mother group can be the result of a greenfield site (Johns, 1993, p. 586), also referred to as skunkworks (Rogers, 2003, p. 149). These are especially supported and enriched groups which are intended to create innovations. Initially, they are often located at the network periphery in order to shield them from other groups' pressure to conform. The other parameters take the following values: $S_{A}=6, S_{N}=4, P_{A N}=P_{N A}=1$.

All simulation runs in this paper were conducted using Vensim DSS 6.1c with Euler integration and $\mathrm{dT}=0.03125$ on a standard PC. For each simulation run, the underlying migration rate is assumed to be equal among all five groups in the organization. Fig. 3 shows the results of five simulation runs which differ with regard to this underlying migration rate. Fig. 3 illustrates that the average adopter fraction over all five groups reacts to an increasing migration rate in a non-linear way. In case the migration rate is only 7.5 percent (graph 5 in Fig. 3), the average adopter fraction reaches an equilibrium of about 22 percent. This is only slightly higher than at the beginning of the simulation, when one out of the five groups consisted only of adopters $\left(M G r_{1}\right)$, an average adopter fraction of 20 percent. A similar behavior can be observed when the migration rate is 10 percent (graph 4 in Fig. 3).

If the migration rate increases to 12.5 percent (graph 3 in Fig. 3), the fraction of adopters migrating from group 1 into group 2 is large enough to convert the nonadopters in group 2. This causes a domino effect in group 3,4, and 5, resulting in the complete diffusion of the innovation throughout the organization. A further increase of the migration rate to 15 percent (graph 2 in Fig. 3) accelerates this diffusion process. However, if the migration rate is 17.5 percent (graph 1 in Fig. 3), all groups reject the innovation. In this case, the adopter fraction within group 1 is not sustainable because too many non-adopters immigrate from group 2 replacing the adopters that migrated from group 1 into group 2. In summary, only a migration rate between 10.1 and 17.1 percent results in a total diffusion of the innovation. Lower migration rates lead to an average adopter fraction of around 20 percent while higher rates cause a complete rejection of the innovation by converting all adopters of the mother group 1 to nonadopters. 


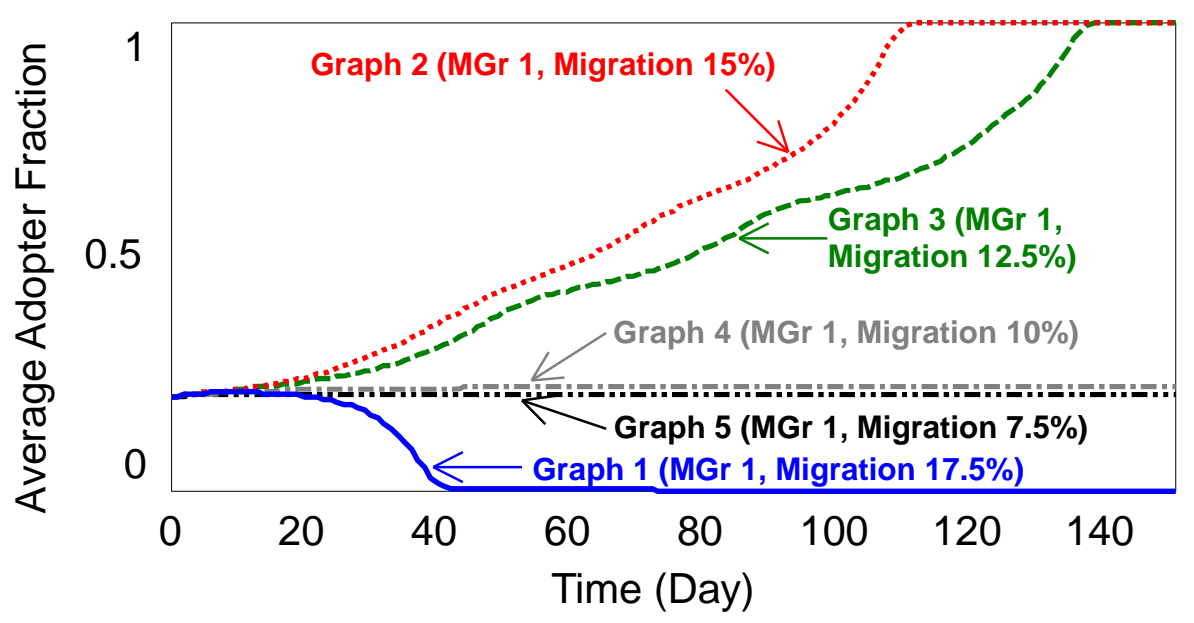

Fig. 3. Effects of different migration rates on the innovation diffusion process with adopters initially situated in group 1 only.

These results are in line with Krackhardt (1997) who refers to this narrow window of opportunity in which an adopter minority wins over a non-adopter majority as the Principle of Optimal Viscosity. Further simulation runs confirm that this principle is surprisingly insensitive to different search intensities, as long as word-of-mouth strength $S_{A}>S_{N}$, and to changes of the conversion probabilities. Since unequal conversion probabilities $\left(P_{A N}>P_{N A}\right.$ or $\left.P_{A N}<P_{N A}\right)$ also cause one camp to be more resistant to conversion than the other, they have a similar effect on the diffusion process. Generally speaking, the window of opportunity is wider, the lower the conversion probability and/or the higher the search intensity of adopters. On the other hand, the window is narrower, the lower the conversion probability and/or the higher the search intensity of non-adopters. Thereby, a lower conversion probability of a camp (i.e., adopter or nonadopter camp) can to some extent compensate for a lower search intensity of that camp and the other way around. Thus, the general behavior of the model would be the same if $P_{A N}<P_{N A}$ instead of $S_{A}>S_{N}$.

The window of opportunity illustrates that too much communication between groups (i.e., a too high migration rate) can be detrimental to the organization-wide diffusion of an innovation. The higher the communication intensity, the greater the mutual reassurance between non-adopter-dominated groups. In this case, the higher number of united non-adopters quickly converts the minority of adopters, even though positive word-of-mouth has a higher impact than negative word-of-mouth $\left(S_{A}>S_{N}\right)$. If the communication intensity between groups is too low, the adopter-dominated group is not able to convert enough non-adopters in the groups it directly communicates with. Only moderately intense communication minimalizes the reassurance effect between nonadopter groups, while maximizing the higher impact of positive word-of-mouth.

The outcome of the diffusion process is sensitive not only to the migration rate but also to the position of the mother group within the chain structure. Fig. 4 illustrates the average adopter fraction as a function of the migration rate when the only difference to the previous simulations is that now group 3 is the mother group $\left(M G r_{3}\right)$ composed only of adopters. The simulations depicted in Fig. 4 show that the window of opportunity for an adopter minority now completely disappears. Ceteris paribus, there is no migration rate which enables the adopters in group 3 to convert all non-adopters in the other four 
groups. In contrast to the mother group being the peripheral group 1 (graph 5 in Fig. 3), the centrally located mother group 3 cannot maintain its adopter majority when the migration rate is 7.5 percent (graph 1 in Fig. 4). Due to the fact that group 3 is connected to two groups instead of to just one, the fraction of emigrating adopters as well as immigrating non-adopters is twice as big. Lowering the migration fraction to 6 percent (graph 2 in Fig. 4) only delays the extinction of adopters in group 3 but cannot prevent it. In case the migration rate is only 4.5 percent (graph 3 in Fig. 4), the conversion of the immigrating non-adopters can compensate for the emigration loss of adopters within group 3 . Therefore, the adopter fraction of group 3 stays close to 100 percent. However, in contrast to the previous simulations depicted in Fig. 3, the emigrating adopter fraction is not large enough to survive within the non-adopter dominated groups 2 and 4, let alone to prevail over the non-adopters there. This results in an average adopter fraction of 20 percent. These findings coincide with Krackhardt (1997) who refers to the increased likelihood and speed of diffusion of a rather isolated mother group as the Principle of Peripheral Dominance.

Besides the Principle of Optimal Viscosity and the Principle of Peripheral Dominance, our model is also capable of reproducing Krackhardt's (1997, p. 196) finding that it is "almost impossible for the non-adopters to retake control of the organization once adopters have dominated it." This so-called Principle of Irreversibility is the result of the assumption that the search intensity of adopters is higher than the search intensity of non-adopters. After introducing the underlying system dynamics model of this paper and after replicating and elaborating on Krackhardt's (1997) main findings, the next section investigates the underlying dynamic processes and behavior modes, which have not been derived from his original algebraic model. We also test management's policies to influence innovation implementation.

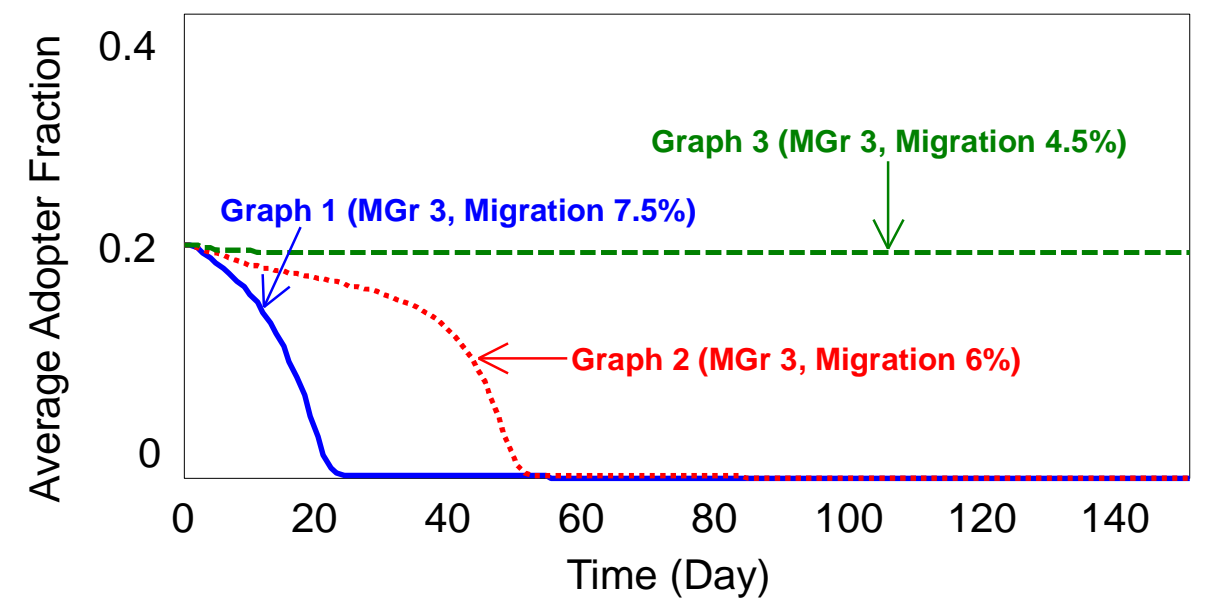

Fig. 4. Effects of different migration rates on the innovation diffusion process with adopters initially situated in group 3 only.

The online supplement contains a further investigation of the potential dynamics within a five-membered chain structure. It shows that the conversion process, which can be characterized as a reinforcing feedback loop, mainly drives dynamics within organizational groups. That is, the higher the adopter fraction of a group, the higher the conversion rate within this group, leading to an even higher adopter fraction. On the other hand, migration processes largely drive dynamics between groups. They can be 
described as balancing feedback loops. Thus, the higher the difference between the adopter fractions of two connected groups, the greater the fraction of adopters migrating from the group with the higher adopter fraction to the group with the lower adopter fraction, which, in turn, decreases the difference between their adopter fractions.

Overall, the simulation runs show that the reinforcing conversion process drives the adopter fraction within a group either towards 1 or towards 0 , depending on whether the adopter threshold has been reached or not. In the cases where an adopter minority (e.g., $M G r_{1}$ ) can stand up to a non-adopter majority and convert all employees, the migration process between the adopter group and a non-adopter group compensates for the conversion process' negative influence within the non-adopter group. In these cases, the fraction of immigrating adopters is bigger than the fraction of converted adopters, resulting in a steady increase of the adopter fraction in the non-adopter group. In the process, the fraction of migrating adopters is large enough to reach the adopter threshold within the non-adopter group, while, at the same time, it is small enough to secure an adopter fraction above the threshold within the adopter group, thereby ensuring a positive influence of the conversion process.

\section{Managerial influence on organizational implementation processes within different organizational structures}

These dynamics not only apply to the examined five-membered chain structure (see Fig. 1 and graph I in Fig. 5). Fig. 5 illustrates other possible network structures whose simulation results, not displayed here, confirm the presence and decisive impact of the elementary dynamics within and between groups. The findings concerning the effects of reinforcing conversion, of balancing migration, and the positive effect of a greater difference between the search intensities of adopters and non-adopters thus hold on a more general level. In this section, we analyze the different network structures in greater detail in order to examine how organizational decision-makers can use the dynamic insights revealed in the previous section to derive more effective implementation strategies. After all, it is often senior management making the decision to adopt an innovation within an organization and being interested in its company-wide diffusion (Rogers, 2003). Hence, this section investigates how senior management can influence these dynamics in order to steer the diffusion process effectively and efficiently. 
(I) Chain/Line

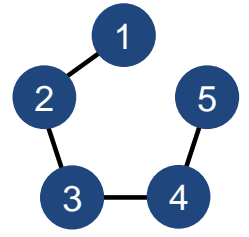

(IV) Ring

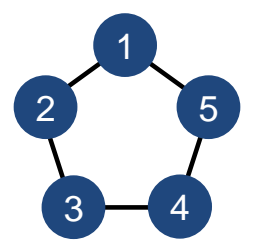

(II) Hierarchy

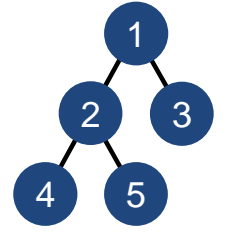

(V) Partially

Connected

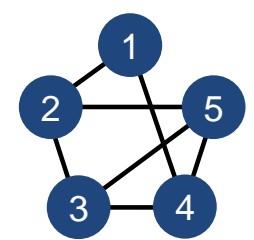

(III) Star

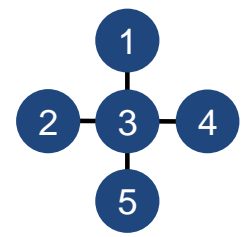

(VI) Fully

Connected

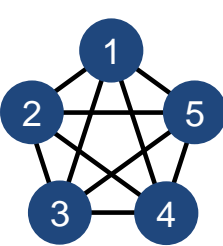

Fig. 5. Six illustrative topologies of network structures using the example of five groups

For this reason, as shown in Fig. 6, we extend the model so that it incorporates senior management's impact on the diffusion process. In contrast to the previous section, we no longer assume that there is an initial mother group within the organization. Instead, we assume that initially all groups consist of non-adopters and that senior management initiates the diffusion of the innovation by changing the targeted degree of diffusion from zero to one at day twelve. Senior management influences certain selected groups (addressed groups) by introducing the innovation to them, by training the respective employees on how to use it, and by exerting normative pressure to actually use it. Senior management's influence on employees' individual adopting decisions has been proven in several studies (Venkatesh et al., 2003; Choi and Chang, 2009; Kim and Kankanhalli, 2009). For example, Choi and Chang (2009) showed empirically that management support significantly improves implementation effectiveness as well as innovation effectiveness by strengthening employees' collective innovation confidence and collective innovation acceptance. 


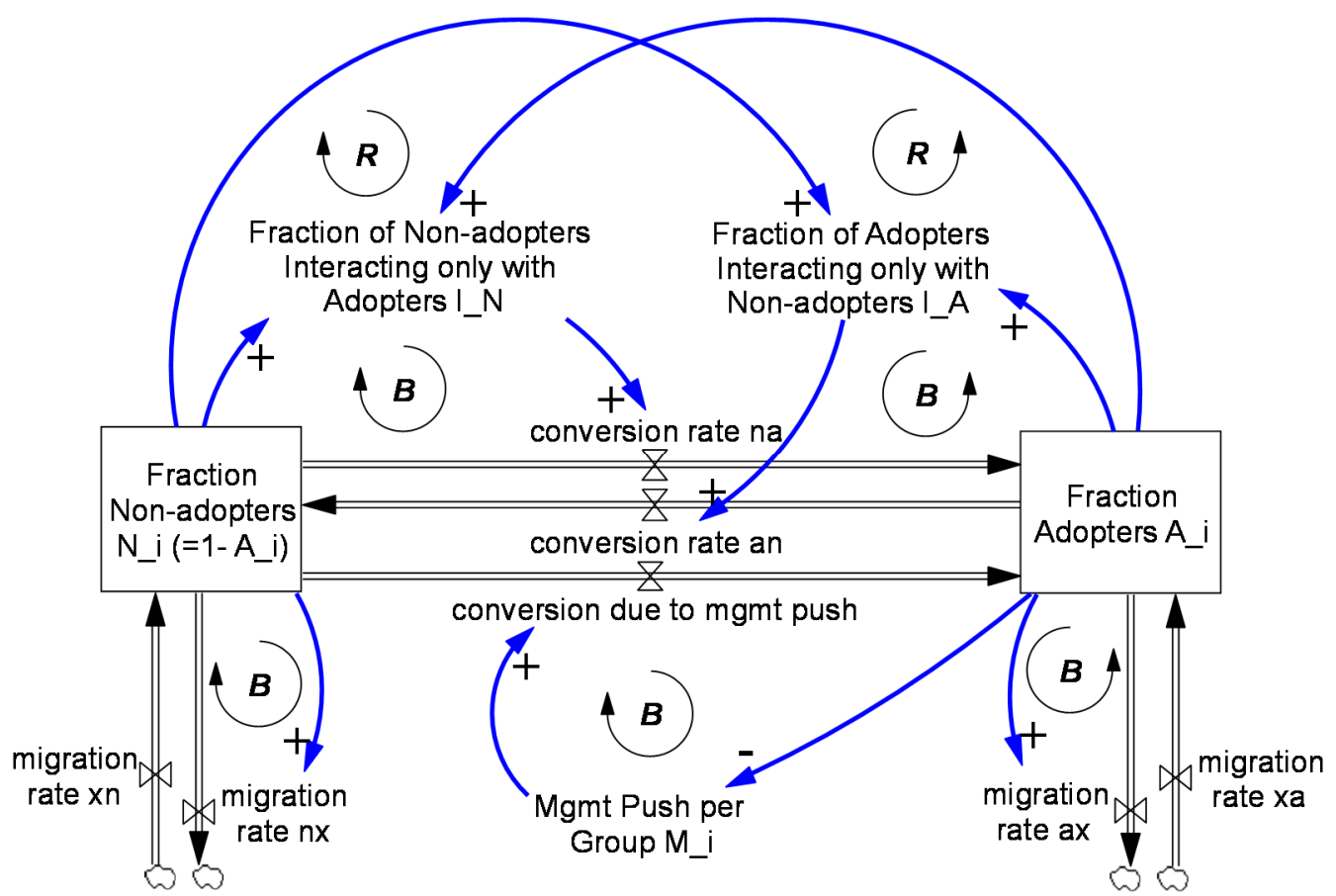

Fig. 6. Simplified stock and flow diagram of the extended system dynamics model (the five organizational groups are modeled with subscripts).

In line with Repenning (2002), we assume that management needs time to develop and implement actions to convert non-adopters and that non-adopters need time to react, acquire skills, and modify their behavior $\left(t_{M}\right)$. We assume that on average these activities take twelve days to become effective. Our sensitivity analysis has shown that decreasing this time constant accelerates diffusion while increasing it hampers innovation diffusion. It is also assumed that the discrepancy between the group-specific degree of diffusion $\left(A_{i}\right)$ and the diffusion degree desired by management equals the amount of pressure senior management exerts on each of the selected groups $\left(M_{i}\right)$. Management's normative pressure $\left(M_{i}\right)$ influences the conversion process within the addressed groups so that the total change in the adopter fraction of a group $i$ per day equals:

$$
\frac{d A_{i}}{d t}=\sum_{j} A_{j} \cdot m_{j i}-\sum_{j} A_{i} \cdot m_{i j}+P_{N A} \cdot I_{N}-P_{A N} \cdot I_{A}+\frac{\operatorname{Min}\left(N_{i}, M_{i}\right)}{t_{M}} .
$$

In the following, we examine which groups senior management should influence to ensure an effective and efficient diffusion throughout the organization. Besides the management-related parameter values already mentioned in this section, all other parameters take on the values of the previous section $\left(S_{A}=6, S_{N}=4, P_{A N}=P_{N A}=1\right)$. By changing the value of the vector addressed groups (see full model documentation in electronic supplement), which describes on what groups senior management exerts pressure, we first examine which groups are most susceptible to management's influence due to their position within the five-membered chain structure (Fig. 1 and graph I in Fig. 5), before generalizing the findings to other network structures. The findings can be reproduced by means of the underlying model. Thereby, the model's interface allows the user to switch between network structures with only a click. The model with all simulation runs and the electronic supplement are available online. 
The simulation results show that senior management's efforts do not result in a complete diffusion if it exerts normative pressure on only one out of five groups. In case senior management influences two groups, there is a window of opportunity for the innovation to diffuse throughout the whole organization only if management influences group 1 and group 2 or their structural equivalent, group 4 and group 5. If management exerts normative pressure on three or more groups, there is always a certain range of the migration rate which enables the complete diffusion of the innovation, no matter which groups the management team influences. Generally, the greater the number of groups being influenced by senior management, the higher the probability that there is a migration rate for which the innovation diffuses throughout the whole organization.

However, resources are often scarce. Therefore, it is important to know what groups and combinations of groups are most susceptible to management's normative pressure and why this is the case. For simplicity, we assume that the accumulated amount of exerted pressure represents the total amount of resources senior management invests to develop and implement actions targeted at creating normative pressure.

In order to identify and reveal the structural characteristics of the most resourceefficient diffusion strategies, we examine those cases where management successfully influences only two or three groups. If two groups are addressed, as mentioned in the previous paragraph, there is only one structurally distinct management strategy within the five-membered chain structure for which the innovation diffuses completely, namely exerting normative pressure on groups 1 and 2 or on groups 4 and 5. In case management influences three groups, there are six structurally distinct strategies, all of them ensuring the complete diffusion of the innovation within all five groups. Thus, in order to outline the key structural elements of the five-membered chain structure, we focus on the following five strategies: strategy A-exerting pressure on groups 1 and 2; strategy B-influencing groups 12 , and 3; strategy $\mathrm{C}$-influencing groups 1, 2, and 5; strategy D-influencing groups 1, 3, and 5; and strategy E-influencing groups 2, 3, and 4 . The following results are also valid if the two omitted strategies - consisting of groups 1, 2, 4 and group 1, 3, 4-were included. Since the innovation diffuses throughout the organization for a range of possible migration rates, the migration rate was chosen that minimizes the diffusion time for the respective strategy, thereby assuming optimality for each strategy regarding migration. The underlying migration rate is 17 percent for strategy A, 24 percent for strategy B, 16 percent for strategy C, 7 percent for strategy D, and 10 percent for strategy E. The left part of Fig. 7 illustrates the development of the average adopter fraction when management employs strategies A to E (indicated by the respective graphs).

Even though strategy A comprises only two groups and requires the most time for the innovation to diffuse completely (graph A in left part of Fig. 7), strategy A is not necessarily the worst of the five strategies when taking into account the invested resources (graph A in right part of Fig. 7). Depending on the management-specific weighting of diffusion time and invested resources, strategy A may well be preferable over all other strategies because strategy $\mathrm{A}$ is most resource-efficient. That is, if the invested resources are much more valuable than a quick diffusion, strategy A (i.e., only exerting pressure on two organizational groups) might be the best choice (right part of Fig. 7).

The simulation results depicted in Fig. 7 show that strategies A and B dominate over all other strategies with regard to resource consumption (graph A in right part of Fig. 7) 
and the diffusion time, respectively (graph B in left part of Fig. 7). Following Krackhardt (1997), it can be argued that the peripheral position of the influenced groups (groups 1, 2 and 3) causes this dominance. However, strategy $C$ exerts pressure on the three most peripheral groups (groups 1,2 and 5) but is only third-best in terms of diffusion time and fourth-best regarding resource consumption (graph C in Fig. 7). Therefore, Krackhardt's (1997) Principle of Peripheral Dominance cannot explain why, for example, strategy B outperforms strategy C.
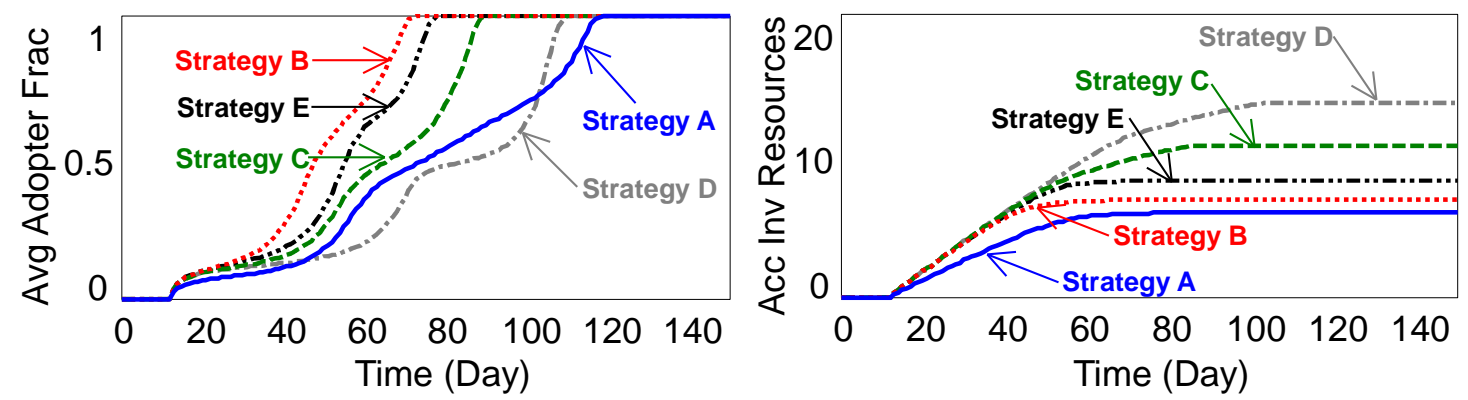

Strategy A : Management pressure on groups 1,2, Migration rate 17\% Strategy B : Management pressure on groups 1,2,3, Migration rate $24 \% \ldots . .$. B......... Strategy C : Management pressure on groups 1,2,5, Migration rate $16 \%---6----$ Strategy D : Management pressure on groups 1,3,5, Migration rate 7\% =-"-D.-" Strategy E : Management pressure on groups 2,3,4, Migration rate 10\% -.....-E-

Fig. 7. Average Adopter Fraction and Accumulated Invested Resources for five different management strategies.

The previous analysis of the diffusion dynamics revealed that migration is a balancing feedback process. That is, the greater the difference of the adopter fractions between two connected groups, the bigger the negative (positive) impact of the migration process on the adopter fraction of the group with the initially higher (lower) adopter fraction. Consequently, the migration process reduces the gap between the two adopter fractions. If senior management influences groups which are connected to each other, the adopter fractions of those groups resemble each other. This, in turn, decreases the migration process' negative effect because the influenced groups support each other by exchanging adopters, thereby limiting the impact of neighboring non-adopterdominated groups on which no pressure is exerted. Thus, strategy B is superior to strategy $\mathrm{C}$ because all three groups are connected to each other. For the same reason, strategy E outperforms strategy D (graphs D and E in Fig. 7). Thus, we find that proximity is an important principle affecting the implementation of innovations.

However, even though strategy $\mathrm{E}$ focuses on three proximate groups, it is inferior to strategy B. These two strategies differ as the latter influences the three connected groups closest to the periphery of the network while the former exerts pressure on the three most central groups. Therefore, it seems that Krackhardt's (1997) principle of peripheral dominance complements the principle of proximity in determining a successful diffusion strategy. Generally speaking, it appears that diffusion strategies focusing on groups which are close to each other and located at the periphery of the network lead to a quicker diffusion at lower costs than strategies which exert pressure on groups which are centrally located and "far away" from each other. However, these findings only relate to communication networks that are organized in a five-membered chain structure (graph I in Fig. 5). In a next step, the derived characteristics of successful diffusion 
strategies (i.e., peripheral location and proximity of the influenced groups) are tested for the other five structures depicted in Fig. 5. For this reason, we quantify the location of a group by calculating its eigenvector centrality (Bonacich, 1972; Ruhnau, 2000;

Bonacich, 2007), whereas we measure the proximity of two groups by determining the geodesic distance between both groups (e.g., Wasserman and Faust, 1997).

The concept of eigenvector centrality is chosen to measure the location of a group within a network because it represents "a weighted sum of not only direct connections but indirect connections of every length. Thus it takes into account the entire pattern in the network." (Bonacich, 2007, p. 555) Hence, the centrality of a group $i$ is higher, the greater number of groups $j$ connected to group $i$, and the greater number of groups to which group $j$ in turn is connected. Consequently, peripherally located groups are characterized by a relatively low eigenvector centrality score. Groups with identical eigenvector centralities have the same influence on the network as a whole and are structural equivalents. For example, group 1 and group 5 in the chain structure have the same eigenvector centrality because they are structural equivalents (Fig. 8). In the following, the eigenvector centralities of groups are based on the Euclidean normalization in order to make them comparable across different network structures (Ruhnau, 2000).

To quantify the proximity between influenced groups, we determine the mean geodesic distance of all pairs between influenced groups. That is, if three groups $i, j$, and $k$ are influenced, the geodesic distances between groups $i$ and $j$, groups $i$ and $k$, and groups $j$ and $k$ are determined and then averaged. The geodesic distance is defined as the length of the shortest path between two groups (Wasserman and Faust, 1997). It equals 1 if both groups are directly connected. If there is no direct connection between both groups, the geodesic distance increases with the number of intermediate groups that connect the two groups along the shortest possible path. With regard to strategy $\mathrm{A}$ in the five-membered chain structure analyzed above, group 2 is directly connected to group 1 and group 3 . However, group 1 and group 3 are only connected to each other via group 2. Therefore, the geodesic distance is 2 between groups 1 and 3 and 1 between groups 1 and 2 and groups 2 and 3. The mean geodesic distance between groups 1, 2, and 3 is hence 4 divided by 3 which equals 1.33. Fig. 8 depicts the eigenvector centralities of all five groups within each of the six different network structures illustrated in Fig. 5. In Fig. 8, the network structures themselves are ordered according to their degree of centralization (i.e., the network centralization index). 


\begin{tabular}{|c|c|c|c|c|c|c|}
\hline & (IV) Ring & $\begin{array}{l}\text { (VI) Fully } \\
\text { Connected }\end{array}$ & $\begin{array}{l}\text { (V) Partially } \\
\text { Connected }\end{array}$ & (I) Chain & (II) Hierarchy & (III) Star \\
\hline \multicolumn{7}{|l|}{ Network Structure } \\
\hline $\begin{array}{l}\text { Network Centralization } \\
\text { Index }\end{array}$ & $0.00 \%$ & $0.00 \%$ & $17.17 \%$ & $51.76 \%$ & $80.29 \%$ & $100.00 \%$ \\
\hline Eigenv. Centrality Group 1 & 0.632 & 0.632 & 0.451 & 0.409 & 0.707 & 0.5 \\
\hline Eigenv. Centrality Group 2 & 0.632 & 0.632 & 0.645 & 0.707 & 0.923 & 0.5 \\
\hline Eigenv. Centrality Group 3 & 0.632 & 0.632 & 0.694 & 0.816 & 0.383 & 1.0 \\
\hline Eigenv. Centrality Group 4 & 0.632 & 0.632 & 0.645 & 0.707 & 0.501 & 0.5 \\
\hline Eigenv. Centrality Group 5 & 0.632 & 0.632 & 0.694 & 0.409 & 0.501 & 0.5 \\
\hline Quickest Strategy (Q) & Groups $1,2,3$ & Groups $1,2,3$ & Groups $1,2,4$ & Groups 1,2,3 & Groups 2,4,5 & Groups $1,2,3$ \\
\hline Peripheral Strategy $(P)$ & Groups 1,2,3 & Groups 1,2,3 & Groups 1,2,4 & Groups 1,2,5 & Groups 3,4,5 & Groups 1,2,4 \\
\hline Mean Geodesic Distance Q & 1.33 & 1 & 1.33 & 1.33 & 1.33 & 1.33 \\
\hline Mean Geodesic Distance P & 1.33 & 1 & 1.33 & 2.66 & 2.66 & 2 \\
\hline $\begin{array}{c}\text { Rank of } P \text { when ranking } \\
\text { all } 10 \text { strategies according } \\
\text { to their diffusion time }\end{array}$ & $1^{\text {st }}-10^{\text {th }}$ & $1^{\text {st }}-10^{\text {th }}$ & $1^{\text {st }}$ & $6^{\text {th }}-7^{\text {th }}$ & $7^{\text {th }}$ & $7^{\text {th }}-10^{\text {th }}$ \\
\hline
\end{tabular}

Fig. 8. Comparison of management strategies across increasingly centralized network structures with senior management exerting pressure on exactly three groups.

Fig. 8 also shows which groups senior management should target in order to ensure the quickest diffusion of the innovation. We compare the quickest strategy (Q) to the strategy which focuses on the three most peripheral groups (i.e., the groups with the lowest eigenvector centralities). It can be seen that the peripheral strategy (P) not only fails to ensure the quickest diffusion for structure I, which was analyzed in detail above, but also for structures II and III. On the other hand, the proximity of the three influenced groups (i.e., the mean geodesic distance between them) also fails to predict the quickest strategy. That is, in case of structure V, a strategy influencing groups 3, 4, and 5 realizes the lowest possible value of the mean geodesic distance, which is 1 . However, focusing on groups 3, 4 and 5 is not the quickest strategy (Q) because these are the most central groups. Thus, the previous suggestions that management needs to consider both centrality and proximity and that it should influence peripheral groups which are proximate to each other also hold for other structures. In the following section, these and other findings of this research as well as their underlying dynamics are summarized and discussed.

\section{Discussion and conclusions}

This paper analyzes the influence of managerial implementation strategies on the diffusion of innovations within intra-organizational networks by means of a system dynamics model. In contrast to Krackhardt's (1997) and many others' work (e.g., Gibbons, 2004; Bohlmann et al., 2010), we do not only examine how input variations influence the output of the model. Over and above, we focus on revealing and describing the inherent dynamics which actually define the output. We alter the model's input and analyze its effect on the output in order to elucidate the underlying dynamics. The model extends common formulations of diffusion processes in system dynamics by explicitly accounting for repeated decisions about the daily use of an innovation, for organizational groups organized in particular network structures, and for management's normative influence on the diffusion process. 
This study finds that senior management should consider the position of organizational groups in the intra-organizational network when deciding which groups to influence. In particular, we analyzed six different network structures to identify structural characteristics that make some groups more susceptible to management pressure than others. In order to realize a relatively quick and resource-efficient diffusion, we found that management needs to follow two rules when deciding on which groups it should exert normative pressure: (i) the selected groups need to be shielded from too many non-supporting groups that are dominated by non-adopters (i.e., influenced groups should be peripherally located); (ii) the selected groups should be close enough to each other to mutually stimulate the level of adoptions in them (i.e., they should be proximate to each other). In many cases investigated here, a peripherally located core of influenced groups proved useful to achieve an efficient intraorganizational innovation implementation. Thus, if management's influence on the diffusion process is considered, Krackhardt's (1997) Principle of Peripheral Dominance is not valid without restrictions. Instead, both, the peripheral location as well as the proximity between influenced groups need to be considered. The earlier is quantified by calculating the eigenvector centrality of each group, while the latter is measured by determining the mean geodesic distance between three influenced groups.

Influenced groups benefit from a peripheral location because they are only sparsely connected to other groups. Thus, the initially negative influence of balancing migration processes is lower than in groups which are characterized by a higher eigenvector centrality. Therefore, management's relative influence on peripheral groups is greater than on more centrally located groups (see also equation 5'). However, groups that are relatively sparsely connected have a rather low influence on other groups. Hence, the adopters of those groups are less capable of persuading the remaining non-adopterdominated groups than they would have been if they had been more centrally located.

Besides the peripheral location, we found that also the proximity of influenced groups plays an important role. Proximate groups support each other by exchanging adopters, thereby limiting the initially negative influence of balancing migration processes. This increases the speed and likelihood that the adopter fraction will rise above the adopter threshold, thereby ensuring that self-reinforcing conversion starts working in favor of the adopter camp. However, this effect is weaker, the greater the geodesic distance between two influenced groups. As illustrated in Fig. 8, neither of the two principles alone-Peripheral Dominance and Proximity - seems to predict the quickest management strategy for all network structures. Instead, our dynamic analysis suggests that both principles need to be considered. Future research could examine under which circumstances one principle is more important than the other.

In summary, our analysis has shown that the persuasive power of non-adopters' negative word-of-mouth can severely impede the diffusion of an innovation by converting adopters into non-adopters within a group and by spreading across groups due to the migration of non-adopters. In addition, we have demonstrated that the choices of senior management can determine the success or failure of an innovation. That is, senior management should carefully choose the groups it influences to ensure a timely and resource-efficient implementation. Lastly, our analyses of different network structures revealed that senior management should also take into account the communication structure between influenced groups when deciding on a strategy. In particular, we have shown that strategies addressing peripherally located and proximate 
groups facilitate the diffusion process. These findings would not have been possible without extending standard diffusion structures by accounting for employees alternating between using and neglecting the innovation, by considering different organizational network structures, and by including management's influence on the diffusion process.

By considering the effects of managerial implementation efforts on the diffusion process, the extended model provides insights which might aid senior management in controlling the diffusion process of an innovation within an intra-organizational communication network. Thereby, the overall focus of this article has been on the communication process within (conversion) and between (migration) groups. We argued that the concept of migration can be interpreted rather broadly by including across-group communication such as initiating a telephone conversation. Therefore, the proposed model is applicable to multiple cases, providing insights into organizational communication networks that are not solely based on physical migration processes between groups.

While the research presented here has been analyzed for its internal consistency and conceptual fit with reality, it faces limitations that future research can address. This study examines only a limited number of different network structures. Even though the presented findings are expected to hold also for other network structures and other network sizes, additional research is required to confirm this. This study can also serve as a step towards a generic rule which identifies the most effective and/or efficient management strategy for each network structure. Further insights can also be generated by relaxing the assumption that all groups are homogeneous and that the ties between them are equally strong. Concerning network connectivity, effects of individual differences, such as differences in employees' social capital (Adler and Kwon, 2002), are considered only at the group level. Within groups, we only account for the average connectivity of an employee.

In addition, model assumptions are based on literature on intra-organizational innovation implementation and diffusion processes. Thus, our findings relate to the intra-organizational adoption of innovations. However, the principles of peripheral location and proximity also resonate with inter-organizational networks and clusters. Related research has moved away from investigating the geographic or spatial proximity between firms towards concepts similar to those employed in this paper (Porter, 1998). These concepts focus on relational proximity which is based on communication processes between firms (Rice and Aydin, 1991; Torre and Rallet, 2005). In a similar vein, the derived findings might be applied to diffusion scenarios on a market level, such as the roll-out of a new product. Therefore, analyzing communication processes and the resulting dynamics appears promising for understanding how organizations innovate on multiple levels. 


\section{Bibliography}

Abrahamson E, Rosenkopf L. 1997. Social network effects on the extent of innovation diffusion: a computer simulation. Organization Science 8(3): 289-309.

Adler PS, Kwon S-W. 2002. Social capital: prospects for a new concept. Academy of Management Review 27(1): 17-40.

Aiman-Smith L, Green SG. 2002. Implementing new manufacturing technology: the related effects of technology characteristics and user learning activities. Academy of Management Journal 45(2): 421-430.

Asch SE. 1963. Effects of group pressure upon the modification and distortion of judgments. In Groups, Leadership and Men: research in Human Relations, Guetzkow H (ed.). Russell \& Russell Inc: New York; 177-190.

Barlas Y. 1989. Multiple tests for validation of system dynamics type of simulation models. European Journal of Operational Research 42(1): 59-87.

Barlas Y. 1996. Formal aspects of model validity and validation in system dynamics. System Dynamics Review 12(3): 183-210.

Barlas Y, Carpenter S. 1990. Philosophical roots of model validation: two paradigms. System Dynamics Review 6(2): 148-166.

Bass FM. 1969. A new product growth for model consumer durables. Management Science 15(5): 215-227.

Berger J, Milkman KL. 2012. What makes online content viral?. Journal of Marketing Research 49(2): 192-205.

Bohlmann JD, Calantone RJ, Zhao M. 2010. The effects of market network heterogeneity on innovation diffusion: an agent-based modeling approach. Journal of Product Innovation Management 27(5): 741-760.

Bonacich P. 1972. Factoring and weighting approaches to status scores and clique identification. Journal of Mathematical Sociology 2(1): 113-120.

Bonacich P. 2007. Some unique properties of eigenvector centrality. Social Networks 29(4): 555-564.

Chen CC, Law CCH, Yang SC. 2009. Managing ERP implementation failure: a project management perspective. IEEE Transactions on Engineering Management 56(1): 157-170.

Choi JN, Chang JY. 2009. Innovation implementation in the public sector: an integration of institutional collective dynamics. Journal of Applied Psychology 94(1): 245-253.

Damanpour F. 1996. Organizational complexity and innovation: developing and testing multiple contingency models. Management Science 42(5): 693-716.

Dean JW Jr, Bowen DE. 1994. Management theory and total quality: improving research and practice through theory development. Academy of Management Review 19(3): 392-418. 
De Dreu CKW, Nijstad BA, van Knippenberg D. 2008. Motivated information processing in group judgment and decision making. Personality and Social Psychology Review 12(1): 22-49.

Donnellon A. 1986. Language and communication in organizations: bridging cognition and behavior. In The thinking organization, Sims H, Gioia D (eds). Jossey-Bass: San Francisco; 136-164.

Dorogovtsev SN, Goltsev AV, Mendes JFF. 2008. Critical phenomena in complex networks. Reviews of Modern Physics 80(4): 1275-1335.

Eagly AH, Chaiken S. 1993. The psychology of attitudes. Harcourt Brace Jovanovich College Publishers: Fort Worth.

East R, Hammond K, Lomax W. 2008. Measuring the impact of positive and negative word of mouth on brand purchase probability. International Journal of Research in Marketing 25(3): 215-224.

Ford JD, Ford LW. 1995. The role of conversations in producing intentional change in organizations. The Academy of Management Review 20(3): 541-570.

Forrester JW, Senge PM. 1980. Tests for building confidence in system dynamics models. In System Dynamics: TIMS Studies in the Management Sciences, 14, Legasto AA Jr, Forrester JW, Lyneis JM (eds). North-Holland: New York; 209 228 .

Frey D. 1986. Recent research on selective exposure to information. In Advances in Experimental Social Psychology, 19, Berkowitz L (ed.). Academic Press: New York; 41-80.

Gary MS. 2005. Implementation strategy and performance outcomes in related diversification. Strategic Management Journal 26(7): 643-664.

Gibbons DE. 2004. Network structure and innovation ambiguity effects on diffusion in dynamic organizational fields. Academy of Management Journal 47(6): 938-951.

Gosselin M. 1997. The effect of strategy and organizational structure on the adoption and implementation of activity-based costing. Accounting, Organizations and Society 22(2): 105-122.

Greenhalgh T, Robert G, Bate P, Macfarlane F, Kyriakidou O. 2005. Diffusion of Innovations in Health Service Organisations: A systematic literature review. Blackwell Publishing Ltd.: Oxford.

Hansen MT. 1999. The search-transfer problem: the role of weak ties in sharing knowledge across organization subunits. Administrative Science Quarterly 44(1): 82-111.

Janis IL, Mann L. 1977. Decision making: A psychological analysis of conflict, choice, and commitment. Free Press: New York.

Johns G. 1993. Constraints on the adoption of psychology-based personnel practices: lessons from organizational innovation. Personnel Psychology 46(3): 569-592.

Karimi J, Somers TM, Bhattacherjee A. 2007. The impact of ERP implementation on business process outcomes: a factor-based study. Journal of Management Information Systems 24(1): 101-134. 
Kim H-W, Kankanhalli A. 2009. Investigating user resistance to information systems implementation: a status quo bias perspective. MIS Quarterly 33(3): 567-582.

Klein KJ, Conn AB, Sorra JS. 2001. Implementing computerized technology: an organizational analysis. Journal of Applied Psychology 86(5): 811-824.

Klein KJ, Sorra JS. 1996. The challenge of innovation implementation. Academy of Management Review 21(4): 1055-1080.

Krackhardt D. 1997. Organizational viscosity and the diffusion of controversial innovations. Journal of Mathematical Sociology 22(2): 177-199.

Lanzolla G, Suarez FF. 2012. Closing the technology adoption-use divide: the role of contiguous user bandwagon. Journal of Management 38(3): 836-859.

Leonard-Barton D, Deschamps I. 1988. Managerial influence in the implementation of new technology. Management Science 34(10): 1252-1265.

Mahajan V, Muller E, Bass FM. 1990. New product diffusion models in marketing: a review and directions for research. The Journal of Marketing 54(1): 1-26.

Mahajan V, Muller E, Kerin RA. 1984. Introduction strategy for new products with positive and negative word-of-mouth. Management Science 30(12): 1389-1404.

Maier FM. 1998. New product diffusion models in innovation management: a system dynamics perspective. System Dynamics Review 14(4): 285-308.

Milling PM. 1996. Modeling innovation processes for decision support and management simulation. System Dynamics Review 12(3): 211-234.

Porter ME. 1998. Clusters and the new economics of competition. Harvard Business Review 76(6): 77-90.

Prislin R, Wood W. 2005. Social influence in attitudes and attitude change. In The Handbook of Attitudes, Albarracin D, Johnson BT, Zanna MP (eds). Lawrence Erlbaum Associates: London; 671-706.

Repenning NP. 2002. A simulation-based approach to understanding the dynamics of innovation implementation. Organization Science 13(2): 109-127.

Rice RE, Aydin C. 1991. Attitudes toward new organizational technology: network proximity as a mechanism for social information processing. Administrative Science Quarterly 36(2): 219-244.

Rogers EM. 2003. Diffusion of Innovations. Free Press: New York.

Ruhnau B. 2000. Eigenvector-centrality - a node-centrality?. Social Networks 22(4): 357-365.

Schulz-Hardt S, Frey D, Lüthgens C, Moscovici S. 2000. Biased information search in group decision making. Journal of Personality and Social Psychology 78(4): 655-669.

Simon HA. 1956. Rational choice and the structure of the environment. Psychological Review 63(2): 129-138. 
Tsai W. 2001. Knowledge transfer in intraorganizational networks: effects of network position and absorptive capacity on business unit innovation and performance. Academy of Management Journal 44(5): 996-1004.

Torre A, Rallet A. 2005. Proximity and localization. Regional Studies 39(1): 47-59.

Venkatesh V, Morris MG, Davis GB, Davis FD. 2003. User acceptance of information technology: toward a unified view. MIS Quarterly 27(3): 425-478.

Wasserman S, Faust K. 1997. Social Network Analysis: Methods and Applications. Cambridge University Press: New York.

Wood W, Lundgren S, Quellette JA, Busceme S, Blackstone T. 1994. Minority influence: a meta-analytic review of social influence processes. Psychological Bulletin 115(3): 323-345.

Ulli-Beer S, Gassmann F, Bosshardt M, Wokaun A. 2010. Generic structure to simulate acceptance dynamics. System Dynamics Review 26(2): 89-116.

Yücel G, van Daalen CE. 2011. Exploratory analysis of the impact of information dynamics on innovation diffusion. Technological Forecasting and Social Change 78(2): 358-372. 